Théologiques

Théologiques

\title{
D’un Dieu tout-puissant à un Dieu partenaire qui s'offre au
} monde

\section{Une lecture processuelle de Gn 2}

\section{Lydwine Olivier}

Volume 19, numéro 1, 2011

URI : https://id.erudit.org/iderudit/1014188ar

DOI : https://doi.org/10.7202/1014188ar

Aller au sommaire du numéro

\section{Éditeur(s)}

Faculté de théologie et de sciences des religions, Université de Montréal

\section{ISSN}

1188-7109 (imprimé)

1492-1413 (numérique)

Découvrir la revue

Citer cet article

Olivier, L. (2011). D'un Dieu tout-puissant à un Dieu partenaire qui s'offre au monde : une lecture processuelle de Gn 2. Théologiques, 19(1), 193-216.

https://doi.org/10.7202/1014188ar

\section{Résumé de l'article}

L'article s'intéresse à la façon dont la théologie processuelle permet de réfléchir à la représentation de Dieu. En effet, le récit de Gn 2 pose la question du présupposé que tout un chacun porte sur Dieu. Loin d'être une réponse toute faite, une et certaine, la théologie processuelle propose de partir à la rencontre d'un Dieu qui s'offre et qui offre un monde en devenir à l'humain. Pour mieux cerner la pertinence d'une analyse processuelle de ce récit, l'article s'intéresse à certaines questions clefs de la théologie qui la sous-tend. Celles-ci portent sur le libre arbitre de l'humain, l'omniscience et la toute-puissance de Dieu, la question du pôle primordial de Dieu comme pôle transcendant, et celle de son pôle conséquent comme signe de son immanence. L'article montre que le récit est une proposition offerte au lecteur de construire sa propre représentation de Dieu, non dans un postulat figé, mais dans un mouvement à-venir, toujours en train de se créer. 


\title{
D'un Dieu tout-puissant à un Dieu partenaire qui s'offre au monde
}

\author{
Une lecture processuelle de Gn 2
}

\author{
Lydwine OLIVIER" \\ Théologie \\ Université de Montréal
}

Dieu est-il le présupposé de l'humain, ou bien l'humain est-il le présupposé de Dieu? Derrière cette question, c'est la notion de représentation que chacun se fait de Dieu qui est en jeu, au cœur de l'inconnaissable qu'il est. Croire ou non à l'existence de Dieu repose sur une représentation, donc un présupposé. Si l'on s'attarde à la question de Dieu à travers les époques et les régions, on se rend compte qu'il n'y a pas une seule et unique représentation de Dieu. Et ce n'est pas tant dû à Dieu qu'à la façon dont les humains en parlent. Peut-on alors dire, non pas comme Descartes «je pense, donc je suis », mais «je pense, donc Dieu existe (ou non)» ? Et comment cette idée peut-elle s'articuler? Une des idées dominantes qui perdure encore dans notre monde offre la représentation d'un Dieu souvent «omnitout » : omnipotent, omniprésent, omniscient. Un choix de représentation qui ne cesse de rendre actuelle la question de la prédestination. Comment articuler ce concept d' «omni» (surtout d'omnipotence) face aux drames de la shoah, aux drames humains auxquels notre monde de communication instantanée et planétaire nous confronte, ou aux drames personnels de chacun, toujours présents. Surtout, comment articuler l'idée de prédestination dans un monde à l'intérieur duquel la religion dominante ne s'y trouve plus omniprésente? Pourtant, le maniement de ces questions ne mène-t-il pas tout droit à deux façons extrêmes de penser Dieu: la mort de

Lydwine Olivier est doctorante en théologie à la Faculté de théologie et de sciences des religions de l'Université de Montréal. Ses champs de recherche portent sur l'exégèse vétérotestamentaire, la théologie processuelle et la psychanalyse lacanienne. 
Dieu, ou sa volonté absolue sur nous? Quel espace ces deux extrêmes laissent-ils encore ouvert?

Il est possible de se poser cette question dans la lecture du second récit la création, car la Bible reste un lieu où de multiples représentations de Dieu s'offrent à nous: "Qui dites-vous que je suis? » $($ Lc 9,20) Je voudrais tenter de réfléchir à cette interrogation en relisant ce récit sous l'angle de la théologie processuelle. Quelle représentation de Dieu ce texte nous offret-il ? Plus spécifiquement, que permet une lecture processuelle d'un texte mythique de la Bible comme Gn 2 ? Ce récit laisse une large part à la création du vivant, là où le premier récit Genèse 1 concerne davantage la façon dont Dieu, pour créer le monde, opère un travail de séparation.

Je commencerai par cerner l'intérêt d'une lecture processuelle de Gn 2 (I). Il sera ensuite question du libre arbitre des entités (II), puis de l'omniscience et de la toute-puissance de Dieu (III). Cela m'amènera à préciser la particularité de Dieu, comme entité ayant deux pôles. De par son pôle primordial, qui lui permet de médiatiser les possibles, on touche à sa transcendance (IV), alors que, de par son pôle conséquent, on touche à son immanence, comme Dieu participant au monde dont il est partie prenante $(\mathrm{V})$.

\section{La théologie processuelle au service de la lecture d'un texte du Premier Testament}

Longtemps méconnue en Europe et dans le monde francophone catholique, la théologie processuelle, ou théologie du process, offre un intérêt particulier: elle cherche à "démontrer que Dieu est scientifiquement et philosophiquement pensable» (Gounelle 2010, 7, se référant à la pensée de Cobb). Pour cette raison, cette démarche, qui «part de la foi pour en dégager la rationalité » (Gounelle 2010, 7), me semble appropriée dans un monde où spiritualité et matérialisme se côtoient.

La théologie processuelle s'est développée à partir de la pensée d'Alfred North Whitehead ${ }^{1}$, pour qui Dieu est une nécessité logique: il est le seul pouvant remplir les trois fonctions essentielles à la bonne marche du monde: il est d'abord celui qui rend possible l'advenue des potentialités. Il est ensuite celui qui permet à la nouveauté d'être introduite, en incitant

1. «Alfred North Whitehead (1861-1947) expressed his belief that science had developed to a stage that demanded a new scheme of ideas in the philosophical realm to reflect the new ideas in sciences. [...] He believed that all things were interconnected and that philosophy should see reality as an organic unity in opposition to science, which tend to isolate them.» (Gnuse 2000, 28) 
l'entité ${ }^{2}$ à choisir ces potentialités (Gounelle 2010,13-14). C'est enfin lui qui favorise l'harmonie et l'unité, dans une cohérence qui lui donne sa valeur. Par conséquent, au lieu d'envisager Dieu comme extérieur au monde, la métaphysique de Whitehead propose Dieu comme entité dans le monde, au même titre que toute autre entité existante, avec comme différence fondamentale ${ }^{3}$ qu'il est le seul à pouvoir médiatiser les potentialités.

La théologie processuelle offre un autre avantage: c'est un courant qui rejoint des intuitions que l'on retrouve aussi dans la pensée juive sur de nombreux plans. En effet, le procès englobe aussi bien le monde et son rapport à Dieu que Dieu et son rapport au monde, dans une profonde interaction. Il rejoint ici la foi hébraïque pour qui Dieu est au cœur du monde, dans un incessant va-et-vient entre Dieu qui appelle son peuple, et se rappelle à lui constamment, mais aussi ce peuple qui, par la rédaction des textes bibliques, dit au monde qu'il reconnaît ce Dieu comme le Dieu d'Israël, ce Dieu devenu au fil des siècles une source identitaire, une source de foi. Cette notion d'interrelation et de dynamisme, au cœur de la foi hébraïque, est aussi au cœur du process: «Nous avons des relations qui créent des réalités, et non des réalités qui créent des relations » (Gounelle 2010, 12). Relire les textes bibliques à la lumière de la théologie processuelle permet ainsi au théologien, mais aussi à tout un chacun, d'actualiser et de répondre de sa tradition confessionnelle. Puisque Beauchamp suggère possible «qu'aujourd'hui une exégèse allégorique soit proposée sans précautions craintives et même sans se chercher de justifications, sachant ne

2. La première occurrence de chaque terme processuel est indiquée en caractères italiques gras, pour alerter le lecteur qu'il s'agit d'un terme spécifiquement processuel. Les termes processuels seront cependant expliqués au fur et à mesure de leur utilisation. Pour Whitehead, est entité actuelle toute réalité, tout moment d'existence, et non pas uniquement tout être vivant. Il les définit comme des "gouttes d'existence ", moments incompatibles avec la notion même de permanence, toujours en mouvement vers une nouvelle synthèse créatrice. Toute " chose " est donc formée de deux parties: d'un côté les moments d'existence passés, passé objectivé appelé data d'héritage, et de l'autre l'«entité actuelle» que l'on pourrait aussi appeler entité-en-train-dedevenir. Le terme "data d'héritage» indique la partie objectivée des entités, constituée de leur passé, qui ne peut plus se modifier, ni évoluer, mais qui sont offertes au monde - et à Dieu, comme le texte de Genèse 2 va nous le montrer —, aux entités actuelles qui peuvent les utiliser pour devenir autre chose, bouger vers un mieux, vers une complexification de ce que chaque entité est déjà devenue.

Pour toute explication complémentaire sur ces concepts, il existe deux lexiques: celui de Cobb (2008) et celui de Parmentier (1968), à la fin de son ouvrage.

3. Parmi d'autres différences fondamentales. 
faire ombrage à aucune autre " (Beauchamp 1987, 107), il me semble que l'on peut tout autant oser une lecture processuelle d'un texte biblique.

L'intérêt de travailler le second récit de la création réside essentiellement dans son côté archétypale « désigné par les commentateurs modernes comme mythe, comme légende ou tradition folklorique » (Alter 1999, 43), texte offert à la com-prébension ${ }^{4}$ de chacun pour réfléchir aux relations entre Dieu, les humains et le reste de la création. En effet, le récit met en scène Dieu, le monde et ceux qui l'habitent, dans sa phase d'émergence. Il part d'un monde in-fini ${ }^{5}$, donc en état de manques, en attente de compléments et de relations, mais riche de potentiels et du désir de Dieu. Vogels, qui applique au texte des principes sémiotiques, relève qu' « un récit est le résultat d'un manque. Si l'objet recherché est là dès le début, il n’y a pas de récit possible. Si la transformation réussit, la fin du récit consistera dans un état positif, le manque sera comblé » (Vogels 1992, 80). Mais de quels manques parle-t-on? Dieu est-il concerné par ces manques? Le récit va nous dévoiler peu à peu comment Dieu, mais aussi les autres entités, vont vivre et chercher à combler ces manques.

À l'orée de notre réflexion, il peut être judicieux de noter que, loin d'apporter une réponse à nos questions, le texte se veut une invitation à réfléchir au désir de Dieu comme proposition au monde. Dans un monde où il est désormais possible de croire que la mort ne relève plus tant du bon vouloir despotique de Dieu que de la conséquence naturelle et nécessaire de la vie ${ }^{6}$, la théologie du procès permet de réfléchir aux tensions entre science et foi que cela fait surgir, en ouvrant la voie à une réflexion sur la façon de les faire vivre ensemble, tout en les distinguant. Gn 2, comme de nombreux autres textes dont la Bible a le secret, offre une opportunité précieuse de travailler, non seulement sur l'action de Dieu sur le devenir de sa création, mais aussi sur le devenir de l'entité divine elle-même. Car le

4. Par ailleurs, et parce que le procès est un lieu d'ouverture aux possibles, un monde " in-fini », je prendrai la liberté d'éclater les mots à certains endroits, car c'est dans cette cassure que le mot peut ouvrir à d'autres signifiants, force de suggestion offerte au lecteur vers d'autres possibles, comme le fait le texte biblique.

5. In-fini: couper le mot permet de mettre l'accent sur l'aspect «sans fin ", mais aussi «non-fini» de ce monde, marque du manque qui le constitue.

6. «La biologie constate [...] que la mort des individus est une des inventions les plus remarquables du processus de l'évolution, aussi bénéfique pour la vie que l'intervention concomitante de la sexualité. [...] Je veux souligner quel renversement de notre représentation de la mort opère cette constatation que la mort des organismes vivants, en même temps que leur reproduction par mode de sexualité, représente un progrès considérable pour la vie.» (Pohier 1985, 152-153) 
récit offre, à qui s'intéresse à la théologie processuelle, un lieu de recherche fécond à travers les interrelations de plus en plus complexes qui se nouent entre chaque proposition de Dieu, la réponse des entités, et la réaction de Dieu en retour ${ }^{7}$.

Je partirai de cette assertion de Boadt, pour qui les auteurs de la Bible avaient «a sharp vision of who God was and how humans were to act in relationship to this God»(Boadt 1998, 351). Le récit de Gn 2 permet-il d'être aussi affirmatif? S'il est vrai que "the narrative is concerned with the reality of God» (Brueggemann 1982, 44), il est aussi réaliste de noter, comme le fait Vogels que, si l'on s'arrête à une lecture littérale des récits de Genèse 2 et 3, «l'image qu'on retient de Dieu n'est pas très stimulante » (Vogels 1992, 77). Il faudra aussi se demander si, comme Westermann l'affirme, la création des êtres humains « is primeval history, and as such not accessible to our understanding»(Westermann 1987, 205). La question est-elle si facilement simplifiable? Formulé ainsi, ce genre de constat oppose une fin de non-recevoir à toute question. Or, qui ne s'est, ne seraitce qu'une fois dans sa vie, demandé d'où il vient? Mais aussi où il va? Peut-on affirmer, sans en discuter, que dans le récit de Gn 2, «the destiny of the human creation is to live in God's world, with God's other creature, on God's terms » (Brueggemann 1982, 40)?

\section{Dieu face au libre arbitre des entités}

Avant d'aller plus loin, et pour que l'on puisse suivre les développements que j'avance, voici la traduction du récit que je propose ${ }^{8}$ :

$4 b$

$5 \mathrm{a}$

$\mathrm{b}$

c

Le jour où Yhwh Dieu fait terre et ciel, aucune pousse des champs n'existe encore sur la Terre, aucune herbe des champs ne surgit encore parce que Yhwh Dieu n'avait pas encore fait en sorte ${ }^{9}$ qu'il $^{\prime}$ pleuve sur la Terre,

7. «En parcourant globalement la série des séquences, on constate une progression constante du nombre des acteurs en interrelation dans l'espace du jardin » (Thériault 1992, 13).

8. Traduction faite à partir du texte hébreu de la Biblia Hebraica Stuttgartensia. La présentation de la traduction, sous forme de retraits, sert à distinguer entre le déroulement de l'action (texte sans retrait), les paroles (retrait et italiques), et les commentaires narratifs (retrait et régulier).

9. Toutes les formes verbales traduites par l'expression «faire en sorte » sont délibérées. Elle traduit le Hiphil, forme verbale hébraïque comportant deux sujets actifs. Pour 
d

$6 a$

b

$7 \mathrm{a}$

b

c

d

$8 \mathrm{a}$

b

c

9a

b

c

$10 \mathrm{a}$

b

c

d

$11 \mathrm{a}$

b

c

$12 \mathrm{a}$

b

$13 a$

b

$14 a$

b

c

$15 \mathrm{a}$

b il fait en sorte qu'il s'installe dans le jardin d'Éden pour le servir et le garder.

16a Yhwh Dieu ordre-donne ${ }^{10}$ à l'humain:

faire ressortir cette forme verbale particulière, ce que les traductions traditionnelles ne font pas, j'ai choisi une expression qui mette aussi en valeur le second sujet.

10. Le verbe צוה (tswh), qui signifie "ordonner, commander, enjoindre», n'existe en hébreu qu'au D/Piel. Cette forme verbale a ici un sens causatif impliquant un sujet actif et un sujet passif, ce que «commander » suggère effectivement. J'ai emprunté le jeu de mot « ordre-donner » à Marc-Alain Ouaknin, car il joue sur les mots « ordre » et "donner", qui apportent une connotation plus persuasive que le verbe comman- 
de tout animal des champs.

b Mais pour un humain, il ne trouve aucune aide comme son faceà-face.

21a Yhwh Dieu fait en sorte qu'un profond sommeil tombe sur l'humain.

b Il s'endort.

c Il prend un de ses côtés

d et ferme la chair à sa place.

22a Yhwh Dieu construit le côté

c

d Il fait en sorte qu'elle vienne vers l'humain.

23a L'humain dit:

der, en lien avec le fait que les verbes utilisés juste après ne sont pas à l'impératif. Par ailleurs, l'expression concerne l'ordre des choses, de l'ordre de celles qui font vivre: « le fait qu'il y ait un ordre indique que les choses sont à mettre à leur place, celle qui leur revient [...]. Pour l'homme, “être ordonné à" signifie qu'il a une place à occuper» (Ouaknin 1999, 29). Le jeu de mots ouvre un espace que l'emploi courant du mot «ordonner» ne permet plus d'entendre.

11. L'expression «il faut que » permet de traduire le cohortatif présent à cet endroit, un ordre que Dieu se donne à lui-même. Le cohortatif est une construction verbale de type volitif, à la première personne du singulier. Au v. 18, nous avons affaire à un cohortatif sans suite, ce qui est correspond à un ordre qui ne souffre aucune restriction. Dieu, à cet endroit, se donne un ordre absolu à lui-même. Pour ce qui traite des différents cohortatifs, et de leur signification, voir David (2005). 


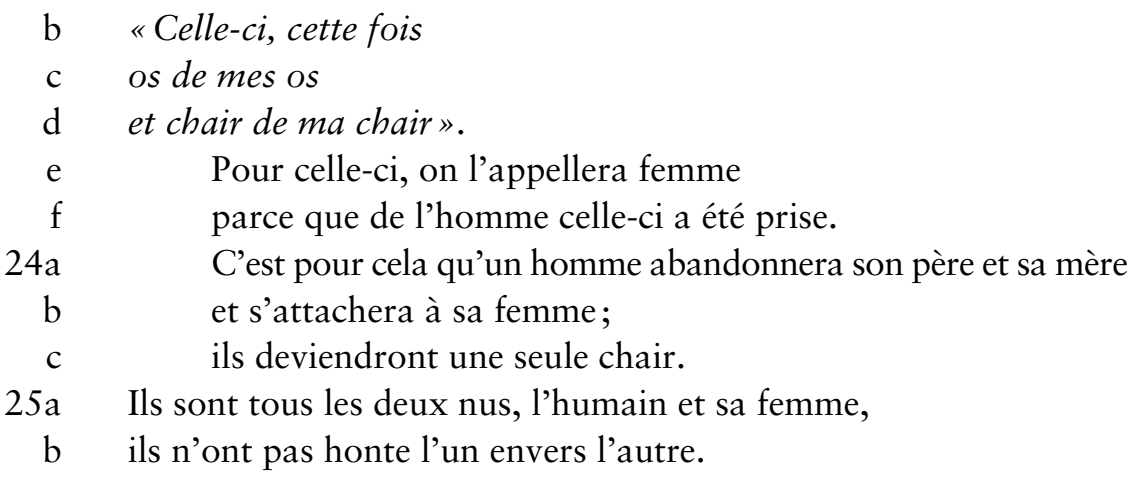

Tous les commentaires perçoivent les manques qui conduisent Dieu à créer. Le sol est vide de plantes, il n'y a pas d'humain pour servir la terre, la pluie est absente (v. 5), et l'eau ne permet pas que la terre boive de façon adéquate (v. 6). Par ailleurs, une fois que Dieu a médiatisé les occasions de vie, les plantes poussent (v. 9a), l'eau abreuve et la terre boit (v. 10-14), l'humain s'installe dans le jardin (v. 15b), les animaux et la femme se déplacent vers l'humain (vv. 19b, 22d). Même le sommeil tombe sur l'humain (v. 21a). Autant d'actions qui sont inscrites en hébreu par des formes au Hiphil, soit une forme verbale comportant deux sujets actifs, Dieu et l'entité. Autant de moments qui montrent que la création n'est pas le jouet de Dieu: les entités sont actives, jouent leur part, comme Dieu qui médiatise des possibles, auxquels la création va ou non adhérer ensuite. On est loin de l'image traditionnelle d'un Dieu seul acteur, et des entités objets, comme le laisse entendre Wenham par exemple, quand il dit qu'aux vv. 5-17, l'humain est complètement passif (Wenham 1987, 50). Thériault est plus nuancé en ce qui concerne l'adam, puisqu'il relève que "Yahvé Élohim assume le rôle de destinateur proposant au sujet [adam] un contrat en rapport avec le jardin et avec les arbres» (Thériault 1992, 27): un contrat implique effectivement deux sujets actifs, et les verbes au Hiphil traduisent ce contrat.

Loin de vouloir, de savoir et d'imposer, Dieu propose, et ne semble pas pouvoir ce que les créatures ne veulent pas. Quand Brueggemann dit que "the woman is also God's free creature » (Brueggemann 1982, 47), il est important d'ajouter à quel point sa réponse et celle de l'humain vont jouer pour que la création vive. Le lieu d'existence passe par l'adversion ${ }^{12}$ des

12. Le mot adversion est un néologisme de Whitehead et signifie, pour une entité, d'accepter la proposition, l'intégrer dans son devenir (ce qui peut aussi correspondre à add version: "ajouter cette version au devenir»). 
entités. En effet, Dieu désire, Dieu propose, Dieu met en route, mais il ne peut pas agir à la place des entités. On mesure cette idée quand «Dieu fait en sorte que l'humain s'installe» (v. 15), quand il s'aperçoit qu'il n'est pas bon que l'humain soit "pour lui-même» (v. 18), quand il crée la femme en réponse au "pas d'aide pour l'humain» (v. 20). Autant de moments où l'autre en face de Dieu devient partie prenante pour la suite de la vie. Le texte nous montre que Dieu est confronté, comme les autres entités, à l'incertitude de nos propres désirs et réactions. Peut-on alors parler, comme le fait Wenham de "God's sovereignty over man » (Wenham 1987, 87) ? Ne doit-on pas nuancer cette affirmation?

Le récit montre plutôt que toutes les entités sont inscrites dans la même aventure, chacune avec sa personnalité et ses choix à affronter. Le texte révèle, encore et encore, que Dieu est lui-même dans une position d'accueil face à la préhension de ses créatures. Il y a un va-et-vient constant entre Dieu et les créatures, une interrelation d'où, petit à petit, émerge la vie, une vie de plus en plus complexe, de plus en plus riche, et qui fait qu'elle est "Vie». Cette vie émerge de cette relation, et non du fait que Dieu «veuille». Thériault note bien ce fait que Dieu ne "veut» pas spécifiquement, quand il pense par exemple qu' "au niveau narratif, on ne peut pas dire que le sujet représenté par l'acteur "Yahvé Élohim" soit principalement en quête d'un “adam pour cultiver le sol” " (Thériault 1992, 23). Pourtant, il ajoute plus loin que «Yahvé Élohim place l'adam dans le jardin en lui assignant un autre contrat ». Encore faut-il se demander s'il s'agit bien d'une assignation. N'est-ce pas plutôt que Dieu est «contraint» de s'adapter à l'humain?

Cette vision vient quelque peu contrarier ce que dit Alter quand il parle de «la tension qui naît de la rencontre entre la volonté de Dieu, en sa conduite providentielle, et de la liberté de l'homme, en sa nature récalcitrante " (Alter 1999, 50). Wenham estime quant à lui que l'humain « doit » son souffle à Dieu, avant d'ajouter plus loin que «contrary to expectation, man is allowed to eat of the tree of life, but not of the tree of wisdom, for that leads to human autonomy and an independence of the creator incompatible with the trustful relationship between man and his maker which the story presupposes» (Wenham 1987, 87). La vision de Wenham soustend que l'humain devrait être d'accord par défaut, ce qui est contraire à la fois à la liberté, que le texte semble impliquer, et à un Dieu qui fait jaillir sans imposer, et que le texte révèle.

En effet, que penser de ce Dieu qui, au v. 20, parle et décide que les animaux ne conviennent finalement pas comme aide-en-face-de-l'humain, 
comme l'ambiguïté du pronom «il » permet de l'envisager ${ }^{13}$ ? Est-ce parce que Dieu évalue mal ses propres propositions? Est-ce parce qu'il n'a aucune idée des conséquences de ses actes? Ou encore est-ce parce que Dieu déciderait pour l'humain de ce qui est bon ou non pour lui ? Adhérer à cette proposition revient à remettre en cause la liberté de l'humain, qui n'aurait alors dans ce cas pas eu son mot à dire. Or, dans une vision processuelle, c'est la position aversative ${ }^{14}$ de l'humain qui conduit Dieu à évaluer que les animaux ne correspondent pas à l'aide devant lui dont l'humain a besoin. C'est la réaction de l'humain qui invite Dieu à réagir une fois de plus. Dieu et l'humain sont étroitement imbriqués dans l'évaluation «pas d'aide pour un humain».

Peut-on dès lors encore parler de la Bible comme d'une «story of how God's word was obeyed or not obeyed in each generation " (Boadt 1998, 349) ? Peut-on encore, à la fin de cette réflexion, dire que Dieu a droit de vie et de mort sur nous? Quelle signification donner à des expressions comme «si Dieu le veut» ou «si Dieu me prête vie»? Qu'avons-nous à répondre face à Dieu, qui, dans ce récit, est le signifiant du mystère de la vie, dans la mise en mots de l'insondable et de l'inconnaissable? C'est pour cela que l'arbre de la connaissance du bon et du mauvais, mis en scène dans Gn 2, a toute sa raison d'être. Dans ce récit, l'humain «devient », et ce devenir inclut la liberté y compris vis-à-vis de Dieu. "L'être humain peut dire non à Dieu, mais par le fait même, il dit non à la vie» (Vogels 1992, 95). Alors, quelle est la place de Dieu dans sa relation à l'humain, si l'on accepte l'idée que Dieu reste dans l'inconnaissance de l'avenir de chaque entité?

13. En effet, le pronom «il» ne permet pas de savoir qui est le sujet réel de cette phrase. Les traductions ont tendance à décider que le pronom «il» vaut pour l'humain: "pour un homme, il ne trouva pas l'aide qui lui fût assortie » $(\mathrm{FBJ})$; «mais pour luimême, l'homme ne trouva pas l'aide qui lui soit accordée » (TOB). Pourtant, le verbe en hébreu ne permet pas de décider. Malgré tout, au niveau syntaxique, on peut légitimement émettre l'hypothèse que ce soit Dieu qui fasse ce constat, car, si cela avait été l'humain, il aurait été raisonnable que l'hébreu ne répète pas le mot humain, mais utilise plutôt la locution pronominale "pour lui ", déjà utilisée deux fois dans ce texte. Le «il» serait alors bien Dieu, qui ne trouve pas d'aide pour l'humain. En tout état de cause, il est préférable que la traduction rende cette ambiguïté, et ne pas décider pour le lecteur.

14. Encore un néologisme: une position aversative, une aversion, revient pour une entité à évaluer négativement une proposition, à ne pas adhérer à une proposition d'une autre entité. 
Nous pouvons trouver la réponse au v. 18 , qui nous introduit à la façon dont Dieu appâte ${ }^{15}$ le monde. En effet, si appâter est possible pour chaque entité, c'est le mode de fonctionnement privilégié de Dieu, toujours à l'affût de stimuler les entités, de les amener vers plus de vie. Dieu, dans ce verset, évalue un manque, et décide d'appâter l'humain: il faut lui donner envie, le mettre en-vie ${ }^{16}$, en route vers ce devenir qui l'attend. De cette évaluation émergera l'autre (et par conséquent aussi son propre devenir), l'aide-en-face-pour-lui, en lieu et place de cette solitude, ce "pour-luimême » mortifère! L'expression, qui signifie solitude, est synonyme d'une forme d'impuissance (Von Rad 1965, 82), mais elle souligne surtout le risque d'un narcissisme, cette toute-puissance égoïste entièrement tournée sur soi qui empêche de considérer ce qui se passe autour, véritable prison relationnelle. Dieu va appâter l'humain avec une nouvelle proposition capable de résoudre cette double problématique. D'un côté, être "une aide ", dans le sens hébreu d'être capable de sauver ${ }^{17}$, et de l'autre, être "en face ", ce qui suppose une notion d'affrontement potentiel. Bref, une entité "debout", capable d'être autant le parfait soutien que le parfait adversaire, sans niveau hiérarchique, sans lien de subordination. L'altérité telle que Dieu la propose, devient alors la façon la plus appropriée de briser la solitude et l'isolement narcissique qui interrompraient le développement relationnel de l'humanité. Dans cette hypothèse, l'accomplissement des Écritures ne se joue pas dans la coercition et le vouloir absolu de Dieu, mais dans la préhension harmonieuse des entités en relation. Le récit est la parabole de ce qui unit si étroitement Dieu et l'humain. L'un sans l'autre peuvent-ils encore exister?

\section{Omniscience ou impuissance de Dieu?}

Si l'on suit les commentaires, Dieu a un projet, Dieu sait ce qu'il veut, ou semble savoir vers quel devenir s'en va sa création, selon son plan. Toutefois, selon d'autres, Dieu fait des essais infructueux, hésite ou doit s’y prendre à plusieurs reprises, selon les expressions glanées çà et là.

15. Appâter est le mot utilisé par Whitehead («lure») pour définir cette volonté sans cesse à l'œuvre chez Dieu de donner envie, d'emmener les entités vers plus de vie, plus de concrescence. On est presque dans l'ordre de la séduction.

16. En-vie: parce que l'envie touche au désir, et met en vie...

17. Ce que rappelle Walter Vogels: «Le mot est rarement appliqué à des humains, mais [...] réfère généralement à Dieu; c'est lui qui est notre aide et notre secours (Ps 33,20)» (Vogels 1992, 83). Il est en cela rejoint par (Wenham 1987, 68). 
Cependant, ces commentaires, qui orientent le lecteur vers une certaine représentation de Dieu, ne confrontent pas leurs conclusions à telle tradition ecclésiale qui présente Dieu comme omniscient, infaillible, qui sait tout d'avance, et dont il n'est jamais envisagé qu'il se trompe. D'ailleurs, comment le pourrait-il, puisqu'il est Dieu ? Comment concilier un Dieu tout-puissant et ce texte, capable de proposer que sa toute-puissance aurait des limites?

Les commentaires parlent du "projet» de Dieu, en un seul mot, ce qui met l'accent sur le fait qu'il a, pourrait-on dire, un plan bien précis en tête ${ }^{18}$, et laisse de ce fait supposer que Dieu savait d'avance comment ses créatures allaient accueillir ses «suggestions ». Ce présupposé va à l'opposé de ce que l'analyse processuelle de ce texte relève. Si, d'un côté, Dieu ne sait pas (ce que le texte semble proposer), et que, de l'autre, Dieu sait tout (ce qu'une certaine théologie propose), alors nous sommes face à ce que le procès appelle un contraste.

En fait, par les notions d'altérité et de contraste, l'herméneutique processuelle considère positivement la possibilité de discorde et de résistance, car elle consent à recevoir l'Écriture non pas comme réservoir de vérités éternelles à répéter aveuglément, mais comme lieu de propositions à évaluer et à valoriser par chaque entité lectrice dans son procès de devenir. (David 2006, 181)

Parce que le texte nous offre cette occasion, prenons le temps de nous y arrêter. Dans l'évaluation que Dieu fait au v. 18 de la solitude de l'humain, les commentateurs ne se demandent pas pourquoi Dieu se fait cette remarque. Seul Thériault indique que «l'acteur divin délibère avant d'agir: “je lui ferai”, [en l'opposant au fait que,] en 2,7, Yahvé Élohim avait entrepris le modelage de l'adam comme si cela allait de soi». Ici, note-t-il, «le faire divin paraît moins naturel ou moins spontané: la performance est envisagée comme une décision réfléchie de s'engager dans un processus lourd de conséquences » (Thériault 1992, 31). Peut-on aller jusqu'à décider que Dieu connaissait ces conséquences? Difficile de dire oui, car le déroulement pas-à-pas du texte révèle un Dieu surpris, dont la réaction a comme

18. Alter parle de la réalisation du dessein de Dieu $(1999,49)$ ou du plan de Dieu (50). Boadt parle des histoires de la Bible qui «deal with human struggles against the limits set by God and our constant search for autonomy to the point of disobedience » (Boadt 1998, 361). Wenham soulève le fait que "despite God's identification of man's need, there is a delay in his provision » (Wenham 1987, 68), laissant entendre que Dieu a compris, mais n'a pas fait... 
point de départ l'arrêt de concrescence ${ }^{19}$ qu'expérimente l'humain. Dieu apprend ainsi de l'humain lui-même que la création ne peut avancer sans l'adhésion de ce dernier, pour qu'il serve et garde la terre.

Dire cela remet en cause ce concept d'omniscience divine, dans le pire des cas. Dans le meilleur des cas, cela montre que Dieu n'avait pas tout prévu d'avance, et qu'il le fait selon des étapes qui ne semblent pas tout à fait préétablies. Par exemple, si Dieu savait tout, n'aurait-il pas dû savoir d'avance que l'humain ne pouvait rester seul? Mais cette hypothèse rend difficilement compréhensible sa prise de parole. En effet, agir aurait suffi, exactement comme il l'a fait depuis le début. Pourtant, ce n'est pas ce qui se passe: Dieu prend la parole ${ }^{20}$ et se donne un ordre ${ }^{21}$. C'est bien que l'heure est grave. À ce moment, Dieu fait l'expérience ${ }^{22}$ de la solitude de l'humain, on pourrait presque dire malgré lui, ce qui remet quelque peu en question le commentaire de Von Rad pour qui «man is created for sociability. God's kindliness sees that it would do man good if a helping creature were given to him as his “opposite” " (Von Rad 1965, 82).

Où se situe alors la puissance de Dieu dans ce récit? Elle n'est certainement pas dans la toute-puissance. Mais si Dieu n'est plus tout-puissant, capable de décider de la vie ou de la mort d'un être, peut-on alors poser la question de son impuissance? En effet, doit-on parler de renoncement ou d'une impuissance quand les entités, une fois en route, sont indépendantes, évoluant selon leurs propres lois, qui ne sont plus alors tout à fait celles de Dieu? Peut-on alors envisager que le texte nous mette, non pas face au pouvoir absolu de Dieu, dans une toute-puissance despotique, mais dans sa volonté absolue et irréductible de faire advenir le monde? Dieu alors,

19. Concrescence: l'entité, en accueillant une nouvelle proposition, s'enrichit de cette nouveauté, et entre dans ce que le procès appelle une étape de concrescence: néologisme formé à partir de con-crescere, grandir avec: il y a une notion de concaténation dans ce concept.

20. Dans la Bible, le choix de faire parler au discours direct un personnage signale l'importance du contenu de ce qui va être dit. Sinon, le rédacteur utilise le discours indirect.

21. Dieu se donne un ordre dans la mesure où la forme verbale employée au v. 18 est un cohortatif direct sans suite, soit la marque d'une contrainte absolue, qui ne souffre aucune exception. C'est un désir profond au point de devenir un impératif que Dieu se donne à lui-même. Robert David propose de traduire ce type de cohortatif par: je vais faire, plus pressant qu'un futur simple, correspondant au I am going to anglais, ou je suis résolu à faire. J'ai préféré traduire ce cohortatif par il faut, afin de mettre en relief cette obligation profonde, et offrir au lecteur une nuance sur la façon dont Dieu se donne au monde (David 2005).

22. La théologie processuelle est très sensible à la question expérientielle de l'entité, qu'elle soit humaine ou divine. Du côté de Dieu, cela correspond à son pôle conséquent. 
pour continuer à agir dans le monde, doit continuer à proposer, tout en sachant que le résultat ne lui appartient pas. La toute-puissance et l'omniscience de Dieu résident certainement davantage là, dans son entêtement insistant, constant et absolu pour encore et encore nous proposer plus de vie, sans pour autant que ces propositions soient celles que l'humain attende ou préhende. Abordé sous cet angle, peut-on, quand la liberté de l'humain entre en conflit avec Dieu, parler de la souffrance de Dieu? Dans ce cas, la souffrance de Dieu n'a-t-elle pas alors un nom, un visage, une existence, qui serait l'autre, en face de soi ?

Dans la même veine, l'arbre du connaître bon et mauvais ne dépend plus tant de la volonté de Dieu que d'une réalité à laquelle, une fois médiatisée, Dieu et l'humain sont confrontés. Cela résout d'une certaine façon la question de cet interdit, imposé pour certains par Dieu. Car, dans ce cas, ce n'est pas d'un commandement dont il est question: Dieu ordre-donne, en ce qu'il énonce un ordre des choses, confronté lui-même à la limite qu'est l'autre, comme il sera confronté à la séduction du serpent ou à la désobéissance de son peuple. Dieu médiatise, mais ne choisit pas d'avance. Vue de cette façon, la remarque de Vogels, «si l'être humain avait pouvoir sur tout, il deviendrait Dieu, ce serait la mort de l'humanité, elle cesserait d'exister» (Vogels 1992, 95), n'est plus valable, car la question n'est pas là. L'analyse processuelle du texte conduit à une réalité: deux entités ne peuvent être identiques. Par conséquent, la question n'est pas que l'humain puisse ou non devenir Dieu, car chaque entité étant unique, l'humain ne pourrait jamais devenir Dieu. Un dieu, peut-être, «comme des dieux » aussi, mais pas "Dieu», puisque Dieu est unique, que l'humain - mais aussi Dieu - le veuille ou non. Ainsi, affirmer alors, comme Vogels le fait, qu' «il n'y a qu'une seule limite: l'être humain ne peut devenir divin» (Vogels 1992, 94) rejoint le raisonnement du serpent en Gn $3^{23}$. Le texte montre plutôt que Dieu n'a pas tout pouvoir. La limite énoncée à l'humain est tout aussi valable pour Dieu, même si cela ne prend pas la même forme, sans possibilité d'y contrevenir.

Le pouvoir de Dieu se trouve dès lors, non dans une toute-puissance réductrice, mais dans le tout amour, la compassion, l'attention et la persévérance irrémédiable, qui, elle, est éternelle, car elle fait partie du temps de Dieu. Comme le dit Vogels, «[Dieu] est un Dieu proche de l'être humain, qui se préoccupe de lui»(Vogels 1992, 96). Ainsi, la citation de

23. Gn 3,5: «Mais Dieu sait que, du jour où vous en mangerez, vos yeux se dessilleront et vous serez comme des dieux, connaissant le bien et le mal» (Bible Osty). 
Beauchamp, à laquelle j'ajoute un terme, pourrait ainsi résumer les lois qui ont émergé de la création: pas que Dieu, "pas tout, pas sans un autre, pas avec le même...» (Beauchamp 1987, 109). Ces lois-là parlent d'une interrelation profonde, nourrissante, vivifiante, où chaque entité a sa place.

C'est pour cela que «God does not intend to be the man's helper. God is helper. But not in this quite secularized presentation» (Brueggemann 1982, 47). Car dans ce récit, il est question de l'amour d'un Dieu pour une créature libre, sans maître ni esclave, et surtout dans une égalité qui exclut la possibilité de dieux qui secourent l'humain. Car la partie serait alors inégale, et l'on retournerait dans la représentation des dieux des païens, à la fois sauveurs, mais aussi capables de se servir des humains comme autant d'objets à leur disposition. La relation que Dieu veut s'établit dans le retrait, non pas de sa présence, mais de son pouvoir divin. Dieu ne fait pas «à la place », Dieu médiatise des possibles: aux entités de poursuivre.

\section{La force du pro-jet comme impulsion primordiale}

L'analyse qui vient d'être faite ne montre pas que Dieu sait d'avance et qu'il a un plan, mais plutôt que, de par sa nature primordiale, il médiatise des possibles. Le résultat de cette médiation ne lui appartient pas, encore moins ce qui émergera de ce que les entités en feront. Même s'il reste, pour chaque événement d'existence, celui qui médiatise encore et toujours les possibles, il n'est pas de son ressort de décider de l'issue de chaque concrescence. Il ne peut donc pas avoir un projet, plan prévu d'avance, mais seulement des «pro-jets", dans un mouvement qui le jette en avant, au cœur de la vie avec tout ce qu'elle contient d'imprévus.

Le texte offre ainsi l'occasion de nous introduire à une représentation de Dieu comme impulsion. On le voit dans le texte: il est l'impulsion donnée au végétal et à la pluie, il est l'artisan modeleur de ce qui est animé, il est à l'origine du souffle de l'humain, il est le bâtisseur de la femme. Cette impulsion, vitale, prend sa source dans son désir de faire advenir la nouveauté, sous forme d'une médiation offerte aux autres entités, dont l'expression la plus absolue est le vivant. Dès le début, le récit nous plonge vers cet «à-venir» irrépressible de Dieu, toujours en lien avec l'existant. Dieu est «pro-jet», sujet superjecté ${ }^{24}$ dans sa création.

24. Sujet superjecté: l'entité (sujet), forte de ce nouveau data, se projette (jet) en avant, dans son devenir avec ce surplus (super). Les trois mots sont importants: «sujet», "super », « jet », car ils forment le tout que l'entité devient à ce moment et transmet pour les devenirs à venir. 
Le texte montre un Dieu fondamentalement préoccupé de sa relation avec nous: l'humain est une donnée que Dieu n'ignore jamais. L'aversion par l'humain d'élever les animaux au rang d'aides-devant-l'humain est une réalité dont Dieu tient compte. Thériault remarque à juste titre que Dieu veut " "voir" et non "entendre" ni "connaître" les noms donnés par l'adam. Yahvé Élohim ne cherche pas en effet à vérifier si les animaux vont être nommés correctement $[\ldots]$ ». Pour autant, peut-on dire comme lui que Dieu «veut tester le comportement même de ce nouveau sujet " (Thériault 1992, 32) ? Je pencherais davantage pour dire que Dieu, entité profondément relationnelle, veut sa création participante: Dieu remet à sa création une part de son devenir, qui est aussi le sien. Dieu apprend en observant la vie et en écoutant la réaction des entités, dans un mouvement continuel de « réaction-adaptation » à ces réactions, pour que son désir de voir advenir la nouveauté et la vie se poursuive.

Car, dans son pôle primordial ${ }^{25}$, Dieu est la seule entité capable de faire en sorte que les objets éternels ${ }^{26}$ puissent éventuellement advenir. Dieu est celui qui fait entrer certains de ces objets éternels dans le monde ${ }^{27}$. Certains seulement, car tous ne peuvent advenir, faute de pertinence ou d'insertion adéquate dans un monde aux possibles limités. Dieu opère là encore une forme de sélection des possibles parmi les possibles, puisque le monde ne pourrait supporter que l'ensemble des possibles s'actualise en même temps. Par son pôle primordial, Dieu devient le médiateur, le vecteur, le lieu qui permet aux objets éternels d'ad-venir, donc de venir en s'additionnant, et ainsi s'introduire dans le monde. Ainsi, ce n'est pas seulement de Dieu, mais avec Dieu que la création du ciel et de la terre peut advenir. C'est par ce pôle primordial que Dieu relie ce qui n'existe pas encore à ce qui existe, ce qui est en puissance à ce qui entre dans la réalité. Ce pôle rejoint d'une certaine façon la transcendance de Dieu, qui, en proposant des actualisations, descend dans le monde, rejoint le monde.

Sans aborder directement la question du mal, qui n'est pas l'objet de cet article, le concept des objets éternels que Dieu propose au monde per-

25. Dieu a deux pôles: dans son pôle primordial, Dieu offre au monde des propositions en lien avec son but initial. Dans son pôle conséquent, Dieu agit et propose en fonction de la réaction des entités aux propositions qui s'offrent à elles, y compris celles de Dieu. Dans ce pôle, Dieu s'adapte aux entités, et propose à partir de cette nouveauté.

26. Objets éternels: potentialités non encore actualisées dans le monde.

27. Mais la réalisation des potentiels dépend des entités, selon qu'elles les préhendent positivement ou non. 
met de réfléchir à la part de responsabilité de Dieu et de chaque entité dans le monde. Dans ce texte, loin de vouloir le mal, Dieu semble vouloir préserver, avertir l'humain de ce qui pourrait lui faire mal et le rendre mauvais. Les entités sont entièrement responsables de leurs actes, qu'elles soient influencées ou non, à leur insu ou non, chacune à leur niveau, Dieu y compris. Vogels le souligne: "Le choix entre la vie et la mort appartient à l'être humain. Dieu lui donne ce que les animaux n'ont pas: la liberté » (Vogels 1992, 95).

Tel est le lourd tribu de la liberté, mais aussi, quel plus beau et précieux cadeau Dieu pouvait-il faire à l'humain ? N'est-ce pas le plus beau et le plus dangereux? Quelle toute-puissance se garde Dieu, quand l'humain devient responsable de sa vie et de sa mort, au-delà même de la simple mort physique? Cela revient à poser la question de la responsabilité de Dieu dans le monde: Dieu est-il celui qui tient le monde dans ses mains, ou est-il un Dieu impuissant, à tout le moins un Dieu dont le pouvoir a ses limites, un Dieu qui se laisse finalement lui aussi surprendre par une vie qui lui échappe tout autant qu'aux autres entités? Oser poser cette question donne à Dieu sa réalité et sa complexité: un Dieu inconnaissable, comme l'est la Vie, qui est aussi et avant tout une aspiration à connaître, à rencontrer, à toucher.

Une lecture processuelle de Gn 2 met l'accent sur Dieu, entité infiniment en expansion, comme on le dit de l'univers, ce qui est différent du fait de «savoir d'avance». La création racontée métaphoriquement en Gn 2 prend cette direction. L'une des composantes du pôle primordial de Dieu est la créativité ${ }^{28}$. Sa présence est avant tout une force créatrice, un désir irréductible de faire advenir, de faire en sorte que la création advienne, dans un surgissement tout aussi irréductible. Peut-on aller jusqu'à dire comme Beauchamp que «c'est par la connaissance-désir que Dieu est en l'homme pour lui faire accueillir un des vivants, y chercher son vis-à-vis, l'écarter, et en laisser venir un autre " (Beauchamp 1987, 112) ? De ce lieu, Dieu est profondément transcendant. Son pôle primordial touche à la verticalité de sa relation avec nous. Les versets de ce récit montrent un Dieu poussé irrésistiblement à proposer, encore et encore, pour que les rencontres aient lieu, que les relations se poursuivent, et que, de ces rencontres, quelque chose de bon en sorte, pour l'ensemble qu'est la création à-venir. Pour cela, Dieu va agir en faisant en sorte que ces occasions poten-

28. La créativité est considérée par Whitehead comme un ultime pour toutes les entités (2006, 138 et $1960,72-73)$. 
tielles, ces potentialités qui n'ont pas encore été médiatisées dans le monde, trouvent un lieu d'existence. Quand Dieu propose, il est transcendant. Mais c'est face à la réaction de l'entité que Dieu va être dans son pôle conséquent. C'est alors qu'on touche à son immanence.

\section{Sollicitude de Dieu et pôle conséquent}

Dans son pôle conséquent, Dieu est une entité dans/avec le monde, qui vit et réagit dans/avec le monde. En cela, la vision de Whitehead, et celle de la théologie processuelle qui en découle, est celle d'un Dieu profondément immanent, impliqué dans le monde comme entité dépendante du monde, de par l'interrelation intrinsèque que, par ce pôle, il entretient avec le monde. Ce qui fait dire à Gounelle ceci: "aussi bien avec la nature primordiale qu'avec la nature conséquente, il s'agit de Dieu en tant qu'impliqué dans le monde. Il y est engagé de deux manières différentes: d'abord comme principe du réel, ensuite comme acteur de l'histoire» (Gounelle 1981, 15).

Le récit apporte deux exemples flagrants du mode d'action immanent de Dieu. Par deux fois, le fil logique apparent du récit est interrompu. Chaque fois, cela correspond à un moment où la concrescence de l'humain est interrompue, où l'on mesure que l'humain n'arrive pas à l'étape de servir la terre, encore moins à celle de la garder. La première fois que le récit semble se bloquer, c'est à la fin du v. 17. Quelque chose ne va pas, ce qui amène Dieu à constater que l'humain est seul. Le second blocage intervient au v. 20, puisque là, on apprend que les animaux n'arrivent pas à remplir le rôle d'aide-devant-lui pour l'humain. Que dire de ces deux arrêts, l'un implicite, l'autre explicite?

Dans les deux cas, Dieu n'est pas seul à décider. Il y a une interaction entre l'humain et Dieu. La décision de Dieu est directement issue de la réaction aversative de l'humain. Dans le premier cas, c'est la parole de Dieu qui met en relief cette interaction. Dieu, en effet, a répondu au premier manque de l'humain au v. 15 : il a vu qu'il lui fallait de la nourriture (des arbres comme nourriture), de quoi désirer (des arbres désirables à voir), il a su qu'il fallait un arbre de la vie et un arbre de la connaissance du bon et du mauvais, et de l'eau canalisée afin qu'il s'installe dans le jardin. Avant ces propositions, l'humain était passif, objet placé dans le jardin, incapable de prendre sa vie en main. Un objet animé, pas encore un sujet désirant. Dieu s'est ajusté.

Au v. 18, Dieu rencontre un nouveau manque, qui semble encore le surprendre et lui faire expérimenter que son désir, que l'humain serve et 
garde la terre, n'est pas rejoint par l'humain. La parole de Dieu répond à un manque implicite, et cette parole sonne comme un signe que Dieu réagit aux conséquences de ses propres propositions. Doit-on aller jusqu'à dire, comme Thériault, que Dieu à ce moment « interprète la situation de l'adam et manifeste de l'insatisfaction" (Thériault 1992, 30)? Il me semble plus exact de dire, comme il le fait, que Dieu « reconnaît un manque dans l'état actuel de l'adam » (Thériault 1992, 30). Sa réaction est exactement inverse à celle qu'il a en Gn 1. Dans le premier récit, pour chaque action qu'il fait, Dieu voit que «cela est bon ». Ici, Dieu ne trouve pas cela «bon». Et ce qui n'est pas bon, c'est que l'humain soit seul. Dieu avait-il prévu ce "grain de sable» ? Tout laisse plutôt penser que Dieu est surpris, et que, attentif à ses créatures, il observe et accompagne la croissance de l'humain. Ce faisant, il se laisse aussi accompagner dans cette croissance et dans celle de sa propre connaissance, qui s'adapte au fil de l'évolution de l'humain. Il a su donner les conditions propices à sa subsistance, à sa structuration saine. Quand il s'aperçoit que, néanmoins, l'humain vit un manque, il mesure que créer du vivant ne suffit pas à le rejoindre. Le texte est résolument un récit du devenir de l'humain, mais aussi du devenir de la relation entre Dieu et l'humain.

Certains commentaires ne s'étonnent pas que le récit ne prenne pas la direction attendue, logique, qui serait que l'humain cultiverait le jardin, s'en occuperait. D’autres, comme Vogels, le signalent, mais en se contentant de dire qu' " un nouveau manque apparaît» (Vogels 1992, 95). Vogels reconnaît même que «Dieu [...] accepte que ce qu'il a fait au début [n'est] pas parfait», et encore que "Dieu accepte l'évaluation négative de l'être humain et tente un deuxième essai» (Vogels 1992, 97). Westermann va jusqu'à parler d'un acte "raté ", en notant de façon quelque peu contradictoire, me semble-t-il, que Dieu a réussi, mais n'a pas réussi ce qu'il voulait (Westermann 1987, 192). Tout aussi intéressante est la réflexion de Beauchamp: «c'est par approximations successives que se réalise [...] le projet de «n'être pas seul ». Il importe que l'homme n'advienne pas d'un coup à son vis-à-vis. On assiste à une sorte d'hésitation, comme si l'hésitation était constitutive de l'homme, et du langage » (Beauchamp 1987, 110), pour ajouter plus loin que «l'hésitation de l'homme est ici précédée, pour ainsi dire, de l'hésitation de Dieu, ainsi présent intensément au temps de naissance du langage, temps où, dans le secret, c'est en réalité Dieu et l'homme qui se cherchent l'un l'autre»(Beauchamp 1987, 111).

Ce constat de manque constitue de ce fait le cœur de la péricope, renforcé par le seul cohortatif ${ }^{29}$ du texte, moment hautement singulier où Dieu

29. Voir note 11. 
se donne un ordre. Syntaxiquement ${ }^{30}$ et processuellement, le sommet du texte n'est donc pas la création de l'humain, mais ce moment où Dieu prend la parole pour créer autre chose, ce moment où Dieu non seulement dit, mais donne vie à son désir, sous la forme d'une contrainte qu'il se donne. À ce moment, Dieu fait une promesse, et cette promesse, c'est d'abord à lui qu'il la fait. Il est alors profondément dans sa nature conséquente. À cet instant, Dieu s'adapte au monde, dans une interrelation où chaque entité, autonome, vient jouer sa part comme partenaire. Non pas avec un projet, mais en se projetant. On est bien plus dans le désir, dans l'espoir, dans le devenir, comme poussée irrépressible, pour lui, pour et avec le monde. Ceci rejoint ce que dit Beauchamp: «l'homme participe de ses lèvres à sa propre création comme à un tâtonnement de Dieu » (Beauchamp 1987, 111), comme le prouve la rupture entre les vv. 17 et 18. Dieu tâtonne parce que l'imprévisible est au rendez-vous, et que l'humain a sa part à jouer. Dieu porte, induit, stimule la vie, réagit à chaque entité, y compris à ce que l'humain dit et fait. Son tâtonnement, mais plus encore son désir, est en fin de compte la preuve et le fruit de la liberté de ses entitéscréatures, comme du choix primordial que Dieu s'est donné de laisser vivre cette liberté, avec en retour l'obligation sans fin de devoir s'y adapter continuellement.

L'avancée créatrice de l'humain est d'avoir dépassé le vouloir de Dieu. On pourrait dire que la créature est allée au-delà de la création initiale. À partir de son data d'héritage — seul, pas «d'aide-devant-lui » —, il amène Dieu à bouger, non plus à partir de son but initial, mais à partir de la rupture dans le déroulement de la création. Dieu, dont la dynamique profonde et perpétuelle est de faire advenir la vie, vouloir la relation et créer, est interpellé: l'arrêt de la concrescence de la vie ne peut lui convenir. Parce qu'elle est inacceptable, impossible, il préhende ${ }^{31}$ cette invitation à s'adapter aux besoins de sa créature et obtenir sa coopération. À partir des data d'héritage, Dieu comprend que ce qu'il a proposé jusqu'alors ne suffit pas. C'est dans ce sens qu'on pourrait dire que la créature a dépassé le désir de son créateur, par la capacité qu'a l'entité "humain» de désirer, lui aussi pourrait-on dire. Et ce désir de l'humain n'est accessible à Dieu que dans le dire (verbal ou non).

30. Le choix fait par le rédacteur de faire parler Dieu pour la seconde fois, en mettant dans sa bouche un cohortatif, doit alerter le lecteur qu'il se passe quelque chose d'extrêmement important à cet endroit.

31. Préhender est encore un néologisme whiteheadien, construit à partir du mot préhension, qui signifie accueillir, faire sien. 
Une fois interpellé, Dieu réagit. Il est essentiel de remarquer que Dieu commence par constater cette solitude et l'impasse qu'elle provoque. Ensuite seulement, il agit. Mais Dieu n'est jamais dans la répétition, qui est une façon de rester dans ce que l'on connaît, exactement à l'inverse de la nouveauté, source de concrescence. La nature de Dieu est dans la nouveauté. À deux reprises, Dieu va créer pour venir à la rencontre de l'humain, mais chaque fois, en y apportant du nouveau. La première fois, il modèle encore à partir du sol, mais sans y mettre son souffle. La seconde fois, on pourrait s'attendre à ce qu'il façonne un autre humain comme aide-en-face, mais il ne le fait pas, parce que ce serait répéter. Il doit trouver de la nouveauté, qui cette fois va résider dans le matériau: l'humain lui-même. Car Dieu est infiniment en concrescence lui-même, comme toutes les entités. La création de l'autre devient une création vitale pour que le désir de Dieu avance. Brueggemann $(1982,51)$ cite sur ce point Trible (1978): "The creation of woman is a second full creation which is necessary to the completion of creation.» Il ajoute: "Woman is the crowning event in the narrative and its fulfillment of humanity », qui vient clore le cercle not God/ not alonelnot other creature (Brueggemann 1982, 47). Dieu est chaque fois mis face à sa créativité du côté de son pôle absolu, mais en raison même de son pôle conséquent. Il répond à ce que le monde et lui-même lui renvoient. Dieu ne sait pas, mais il est toujours en désir que la vie advienne, et en réaction face à tout ce qui est en arrêt de concrescence. Le désir de Dieu peut se voir comme une esquisse, une idée avec de vagues contours, une silhouette, mais aucunement comme quelque chose de plus déterminé.

\section{Conclusion}

L'analyse processuelle de ce récit a permis de s'intéresser à la représentation de Dieu. Si, selon Couturier, «l'idée que l'on se fait de Dieu a des répercussions immédiates sur l'idée que l'on se fait de l'homme» (Couturier 1985, 84), il me semble que l'inverse est tout aussi vrai, peut-être même encore plus. Gn 2 révèle un Dieu transcendant, parce que lui seul est capable d'apporter au monde ce qui, sans lui, ne pourrait advenir. Pourtant, si rien en dehors de lui ne peut advenir, cela ne suffit pas. Dans ce récit, Dieu est tout-puissant, en ce sens qu'il est un médiateur essentiel, incontournable. Mais sa limite réside bel et bien dans l'existence de chaque entité. C'est par et grâce à son pôle conséquent que Dieu fait face et remédie à une certaine impuissance, cette impossible toute-puissance, l'impensable omniscience. Dieu, dans ses deux pôles, est offrande qui se veut avec et non au-dessus du monde qu'il veut voir ad-venir. 
Le texte montre une recherche têtue, compatissante et attentive de Dieu envers ses créatures, ce que relève fort bien Wenham à sa façon: "God worked until all man's needs were satisfied. The God of Genesis is totally concerned with man's welfare. Man is to be more than a tiller of the ground; his need is for companionship, a lack which the creator is anxious to fill» (Wenham 1987, 53). Dieu propose et c'est la préhension qu'en font les entités qui fait d'eux, à leur tour, des entités partie prenante de leur destinée. Le mot destinée n'est pas alors à prendre dans le sens de pré-destination, non pas comme une destination prévue d'avance, mais bien dans le sens d'un à-venir là où la vie emmène, qui se construit dans une interrelation constante entre les entités, entre elles, et avec Dieu.

La création de Dieu est en route, dans un mouvement qui ne se dément pas, mais dont personne, pas même lui, ne saurait dire ce qui va en advenir, rendant chacun intensément responsable de son désir et de ses actes, comme autant de lieux où la création peut vivre, ou mourir. Peut-on dès lors poser cette question: l'accomplissement des Écritures se joue-t-elle dans la coercition et le vouloir de Dieu, ou dans la préhension harmonieuse des entités en relation? Il faut, pour répondre à cette question, garder en mémoire qu'harmonieux n'est pas synonyme de calme plat, mais bien de confrontation et de face-à-face, nécessaires, eux aussi vivifiants, sources et preuves que les entités continuent à grandir, vers une satisfaction personnelle qui emmène le monde vers la pleine satisfaction, mais dont on peut, voire dont on doit, douter qu'elle soit un jour fixée à jamais...

Ainsi, derrière cette analyse, c'est bien une représentation de Dieu que le récit de Gn 2 permet de construire, et que, à titre de lecteur, nous avons à préhender. Cette proposition est orientée sur le fait que rien n'est décidé d'avance, ni par Dieu, ni pour Dieu, mais que la vie se construit de la préhension des possibles par chaque entité, y compris par Dieu, mais non uniquement par lui. Avec lui, par lui et en lui, mais non sans les autres. Dans Gn 2, l'altérité est au cœur de Dieu, comme limite et infini. L'éternité ne se jouerait-elle pas plutôt de ce côté, du côté de l'autre?

\section{Références}

Alter, R. (1999), L'art du récit biblique, Bruxelles, Lessius (Le livre et le rouleau, 4).

Beauchamp, P. (1987), «La création des vivants et de la femme. Lecture allégorique de Gn 2: 15-24", dans La vie de la Parole, de l'Ancien an Nouveau Testament. Études offertes à Pierre Grelot, Paris, Desclée, p. 107-120. 
Biblia Hebraica Stuttgartensia (1967), Stuttgart, Deutsche Bibelgesellschaft. Bible de Jérusalem (FBJ) (1990), Paris, Cerf.

Bible, traduction oecuménique (TOB) (2004), Paris, Cerf.

Bible Osty (1973), Paris, Seuil.

Bondt, L. (1998), «Genesis », The International Bible Commentary, Collegeville, The Liturgical Press.

Brueggemann, W. (1982), Genesis: A Bible Commentary for Teaching and Preaching, Atlanta, John Knox Press (Interpretation).

Совв, J. B. J. (2008), Whitehead Word Book. A Glossary with Alphabetical Index to Technical Terms in Process and Reality, Claremont, P\&F Press.

Couturier, G. (1985), «La mort en Mésopotamie et en Israël: phénomène naturel ou salaire du péché? ", dans A. Charron, G. Couturier et G. Durand, dir., Essais sur la mort, Montréal, Fides (Héritage et projet 29), p. 51-98.

DAvid, R. (2005), «L'analyse syntaxique, outil pour la traduction biblique: le cas des cohortatifs", dans R. David et M. Jinbachian, Traduire la Bible hébraïque. De la Septante à la nouvelle Bible Segond, Montréal, Médiaspaul (Sciences bibliques 15), p. 275-318.

- (2006), Déli_l'ÉCRITURE. Paramètres théoriques et pratiques d’herméneutique du procès, Montréal, Médiaspaul (Sciences bibliques 17).

Ford, L. S. (1978), The Lure of God, Philadelphie, Fortress Press.

Gnuse, R. K. (2000), The Old Testament and Process Theology, St-Louis, Chalice Press.

Gounelle, A. (1981), Le dynamisme créateur de Dieu: essai sur la théologie $d u$ process, Paris, van Dieren.

Gounelle, A. (2010), Le dynamisme créateur de Dieu: essai sur la théologie $d u$ process. Nouvelle édition revue et augmentée, Paris, van Dieren.

Ouaknin, M.-A. (1999), Les dix commandements, Paris, Seuil.

Parmentier, A. (1968), La philosophie de Whitehead et le problème de Dieu, Paris, Beauchesne.

Pohier, J. (1985), Dieu fractures, Paris, Seuil.

Thériault, J.-Y. (1992), "Le parcours de l'Adam dans le jardin », Sémiotique et Bible, 67, p. 13-36.

Trible, P. (1978), God and the Rhetoric of Sexuality, Philadelphie, Fortress.

Vogels, W. (1992), Nos origines: Genèse 1-11, Ottawa, Novalis. 
Von Rad, G. (1965), Old Testament Theology, New York, Harper \& Row.

Wenham, G. J. (1987), Genesis 1-15, Waco, Word Books (Word Book Commentary 1).

Westermann, C. (1987), Genesis 1-11: A Commentary, Minneapolis, Augsburg Publishing House.

Whitehead, A. N. (1960), Process and Reality, an Essay in Cosmology, New York, Harper \& Row.

\section{Résumé}

L'article s'intéresse à la façon dont la théologie processuelle permet de réfléchir à la représentation de Dieu. En effet, le récit de Gn 2 pose la question du présupposé que tout un chacun porte sur Dieu. Loin d'être une réponse toute faite, une et certaine, la théologie processuelle propose de partir à la rencontre d'un Dieu qui s'offre et qui offre un monde en devenir à l'humain. Pour mieux cerner la pertinence d'une analyse processuelle de ce récit, l'article s'intéresse à certaines questions clefs de la théologie qui la soustend. Celles-ci portent sur le libre arbitre de l'humain, l'omniscience et la toute-puissance de Dieu, la question du pôle primordial de Dieu comme pôle transcendant, et celle de son pôle conséquent comme signe de son immanence. L'article montre que le récit est une proposition offerte au lecteur de construire sa propre représentation de Dieu, non dans un postulat figé, mais dans un mouvement à-venir, toujours en train de se créer.

\section{Abstract}

The article focuses on how process theology allows us to conceive the representation of God. The narrative of Gn 2 questions the assumption everyone has about God. Far from providing a one and definite ready-made answer, process theology offers to go and meet a God who offers himself as well as offers a world-to-come to humans. To better understand the relevance of a process analysis of this text, the article focuses on some key issues of the theology that underlies it, such as human free will, omniscience and omnipotence of God, the question of the primordial nature of God as his transcendent pole, and his consequent nature as a sign of his immanence. The article shows that the narrative gives the readers the opportunity to build their own representation of God, not in a static assumption, but in a movement to come, always to be created. 